\title{
EXCHANGE INTERACTION AS A SOURCE OF REAL SPACE PAIRING IN HIGH TEMPERATURE SUPERCONDUCTORS AND HEAVY FERMIONS: A BRIEF OVERVIEW
}

\author{
J. SPALEK \\ Institute of Theoretical Physics, Warsaw University \\ Hoża 69, 00-681 Warszawa, Poland
}

\begin{abstract}
We characterize the principal difference between the pairing induced by the kinetic exchange interactions for the Fermi-liquid and spin-liquid phases. In the case of hybridized electrons the Kondo-exchange induced pairing is invoked, whereas for a single narrow-band case kinetic exchange is responsible for the spin singlet pairing. The former interaction is applied to heavy-fermion systems, whereas the latter is employed to the high temperature superconductors. The current situation concerning the applicability of our theoretical models is briefly and critically assessed.
\end{abstract}

PACS numbers: 71.28.+d, 75.10.Lp, 74.70.Vy

\section{Introduction}

The aim of this paper is to overview in the simplest terms the difference between the real space pairing among electrons in the cases with Fermi-liquid and statistical-spin-liquid normal states chosen as reference states. The discussion is related to the question of the nature of the superconducting state in heavy-fermion and high- $T_{\mathrm{c}}$ systems. Each of these systems can be regarded as a metallic state close to the corresponding localized Mott state: rare-earth (or actinide) metal with localized moments and the Heisenberg antiferromagnet, respectively.

Heavy-fermion systems are regarded in the low temperature $(T<10 \mathrm{~K})$ limit as an almost localized and strongly anisotropic Fermi liquid composed hybridized atomic ( $4 f$ or $5 f$ ) and itinerant $(d-s)$ states forming very heavy quasiparticles. The high-temperature superconductors containing $\mathrm{CuO}_{2}$ planes are considered as either Fermi liquid [1] or as quantum [2] or statistical [3] spin liquids. In this paper we provide the arguments to the latter [4-7].

By real space pairing we uriderstand the pairing induced by the kinetic exchange interactions among strongly correlated electrons in a narrow band [8], which reduce in the Mott insulating limit to the Anderson antiferromagnetic exchange [9]. Also, this interaction in the case of hybridized metallic systems takes the form 
of the Kondo exchange [10], which in the localized-moment limit reduces to the Schrieffer-Wolff form [11]. Both of these interactions are discussed below. The hybrid pairing induced by the Kondo exchange leads to a simple characterization of a paired state for heavy fermions, whereas the kinetic exchange in a two-dimensional spin liquid provides basic features of a high-temperature superconductor [12].

\section{Real space pairing in a narrow band}

The concept of real space pairing was invoked by Anderson $[2,13]$ who proposed to represent the antiferromagnetic exchange interactions via spin-singlet pair operators. As a starting point in that approach one takes the Hubbard Hamiltonian for strongly correlated electrons in a narrow band transformed [10] to the form in which the double occupancies on the same site have been projected out and an intersite spin-singlet pairing included explicitly, namely

$$
H=\sum_{i j \sigma}^{1} t_{i j} b_{i \sigma}^{\dagger} b_{j \sigma}-\sum_{i j k} \frac{2 t_{i j} t_{k j}}{U} \tilde{B}_{i j}^{\dagger} \tilde{B}_{k j} .
$$

The first term represents the band energy with $t_{i j}$ being the hopping integral, and $b_{i \sigma}^{\dagger} \equiv a_{i \sigma}^{\dagger}\left(1-n_{i-\sigma}\right)$ is the creation of an electron on site $i$ and with spin $\sigma$ provided no other electron is present in the same state. The second term represents second order processes $\sim t^{2} / U$, where $U$ is the magnitude of intraatomic Coulomb interaction, and

$$
\tilde{B}_{i j}^{\dagger} \equiv \frac{1}{\sqrt{2}}\left(b_{i \uparrow}^{\dagger} b_{j \downarrow}^{\dagger}-b_{i \downarrow}^{\dagger} b_{j \uparrow}^{\dagger}\right)
$$

is the singlet pair creation operator in real space, i.e. on the pair of sites $(i, j)$. Note that the second term contains both pair binding (the part $\sim \tilde{B}_{i j}^{\dagger} \tilde{B}_{k j}$ ) as well as the pair hopping from the pair of sites $(k j)$ onto $(i j)$ for $k \neq i$ (the three-site part [14]).

This model is difficult to solve for an arbitrary band filling, partly because the one-particle operators $\left\{b_{i \sigma}\right\}$ do not obey the fermion anticommutation relation and the pair operators $\left\{\tilde{B}_{i j}\right\}$ do not obey the boson commutation relations. Additionally, the two terms in (2.1) do not commute with each other and hence, the single-particle and pair motions are mutually connected.

The simplest insight into the nature of electron states for the model (2.1) is obtained by noting that for $n \rightarrow 1$ the expectation value $\left\langle b_{i \sigma}^{\dagger} b_{j \sigma}\right\rangle$ vanishes and therefore, only the pairing part with $i=k$ survives. In this limit (the Mott-insulator limit) the intersite interaction reduces to the Anderson kinetic-exchange form, since

$$
\tilde{B}_{i j}^{\dagger} \tilde{B}_{i j} \equiv-\left[\boldsymbol{S}_{i} \cdot \boldsymbol{S}_{j}-\frac{1}{4}\left(\sum_{\sigma} b_{i \sigma^{\prime}}^{\dagger} b_{i \sigma}\right)\left(\sum_{\sigma^{\prime}} b_{j \sigma^{\prime}}^{\dagger} b_{j \sigma^{\prime}}\right)\right] .
$$

The principal question at this point is whether the Mott insulator in two dimensions can be analysed starting from the Néel state and subsequently including the fluctuations [15], or if one has to start from a novel, resonating-valence bond (RVB) state. In the former case the order parameter is $\left\langle S_{i}^{z}\right\rangle \equiv \frac{1}{2}\left(\left\langle b_{i \uparrow}^{\dagger} b_{i \uparrow}-b_{i \downarrow}^{\dagger} b_{i \downarrow}\right\rangle\right)$, 
whereas in the latter it is $\Delta_{i j} \equiv\left\langle\tilde{B}_{i j}\right\rangle$. In the RVB case it evolves continuously into a paired metallic state of the BCS type and has transition temperature $T_{\mathrm{c}}$ maximal for $n=1$, a feature not observed experimentally. Instead, the superconducting state disappears, the metal-insulator transition takes place and the antiferromagnetic insulating (AFI) state sets in [16]. The AFI state for $n=1$ is well described by the Heisenberg model, and the AF ordered state has a 3 -dimensional character.

One-hole states for the model (2.1) have been treated extensively [17]. Most of the features of these self-trapped states have not been as yet tested experimentally. Also, the nature of the ground state at finite doping and in particular, the transition from the doped Mott insulator to the superconducting metal has not been determined so far, neither theoretically nor experimentally.

\subsection{Fermi liquid approach}

The early treatments of Hamiltonian (2.1) resorted to a Gutzwiller treatment with the second term in (2.1) added after projecting the double occupancies in direct space. In effect, one obtains the starting Hamiltonian in the form [14]:

$$
H=\sum_{i j \sigma}^{\prime} \Phi_{\sigma} t_{i j} a_{i \sigma}^{\dagger} a_{j \sigma}-\sum_{i j k} \frac{2 t_{i j} t_{k j}}{U} B_{i j}^{\dagger} B_{k j}
$$

where now $\Phi_{\sigma}=(1-n) /\left(1-n_{\sigma}\right)$ is the band narrowing factor, $n_{\sigma} \equiv\left\langle a_{i \sigma}^{\dagger} a_{i \sigma}\right\rangle$, $n=n_{\sigma}+n_{-\sigma}$, and now

$$
B_{i j}^{\dagger} \equiv \frac{1}{\sqrt{2}}\left(a_{i \uparrow}^{\dagger} a_{j \downarrow}^{\dagger}-a_{i \downarrow}^{\dagger} a_{j \uparrow}^{\dagger}\right)
$$

is the pair creation operator for fermions with opposite spins (note that the projection present in (2.2) is absent now). In the form (2.3) the starting Hamiltonian represents a Fermi liquid with real space pairing. The model, when solved in mean-field approximation of the Bardeen, Cooper and Schrieffer (BCS) type leads to a nonvanishing value of the superconducting gap $\Delta_{k}$ and a nonzero value of the transition temperature $T_{\mathrm{c}}$ in the limit $n \rightarrow 1$ [18], which corresponds to the Mott insulator. This is clearly a nonphysical result. The situation is not improved by showing that with $n \rightarrow 1$ the antiferromagnetic state is energetically favored, since any resonating-valence bond (RVB) state of the Mott insulator cannot have the same properties as the BCS state. Therefore, the nonvanishing gap for the Mott insulator demonstrates that a simple BCS theory is inapplicable to the almost localized electrons; this is because it relies on the assumption that a stable Fermi liquid (FL) state exists and descibes the normal state of the system. In the next section we introduce the concept of statistical spin liquid (SSL) and later compare the FL and SSL states.

\subsection{Statistical spin liquid in two dimensions}

Electrons in narrow band systems form a correlated quantum liquid, by which we mean the state for which the average band energy $\left|\left\langle\varepsilon_{k}\right\rangle\right|$ per particle (calculated in local-density or related approximation) is comparable or smaller than the magnitude $U$ of the intraatomic interaction energy required to change the occupancy 
of atom by unity relative to the equilibrium configuration. The question is then how to describe the situation in which we start from a well-defined atomic configuration rather than from an electron gas state as the reference state (they belong to separate universality classes). For that purpose we have proposed [3-7] the concept of statistical spin liquid which bears its origin from the Hubbard split-band picture [14] of strongly correlated electrons. In the Hubbard subband picture [19] a single band of quasimomentum (k) states with spin $\sigma$ states is split into two halves: the lower has the weight $\left(1-n_{-\sigma}\right)$, whereas the upper has the weight $n_{-\sigma}$, where $n_{-\sigma}=\left\langle n_{i-\sigma}\right\rangle$ is the average spin $(-\sigma)$ site occupancy. In the limit $n \rightarrow 1$ each subband contains $N$ states: the lower contains only singly-occupied (and empty) states, while the upper distant by amount $\sim U$ comprises the excited doubly occupied configurations. We have ascribed the quasiparticle states to the partially filled subbands by postulating that in the lower subband the doubly occupied $|k \uparrow \downarrow\rangle$ states are excluded from the physical space, whereas the upper subband contains only $|\boldsymbol{k} \uparrow \downarrow\rangle$ states. In this way the volume of the Fermi surface for electrons in the lower subband is doubled with respect to that for FL case. In other words, the Luttinger theorem stating the Fermi volume independence on the interaction magnitude is violated. We connect this violation to the presence of the Mott transition as a true phase transition defining the borderline between the two (FL, SSL) universality classes.

Before turning to the quantitative description of the statistical spin liquid we mention the problem of the SSL stability. It is well known [20] that the Hubbard model in one spatial dimension and for $n=1$ has an insulating state as a ground state for an arbitrary $U>0$. For large number of dimensions $(d \rightarrow \infty)$ the phase diagram [21] is similar to that obtained in the Gutzwiller approach extended to nonzero temperature [22]. In view of these results, the existence of the Mott transition for any $d$ is beyond question. The fundamental question is then what is the nature of state with small number $\delta \equiv 1-n$ of holes in the Mott insulator, as represented by the Hubbard model above the localization threshold. We postulate that it is the statistical spin liquid if only the hole states are not self-trapped in some sort of spin polaron state [23]. This liquid is of metallic character and is assumed to become a stable state at finite doping. A support for such claim comes from the recent work of Anderson [24] who shows that for a two-dimensional system with a Fermi surface the scattering amplitude $f_{k \uparrow k^{\prime} \downarrow}$ is divergent for two particles with $k=k^{\prime}$. Hence, the states $|k \uparrow \downarrow\rangle$ should be projected out from the physical space. It has been shown [16] that the SSL state evolves continuously into the Mott insulator as $\delta \rightarrow 0$. The validity of the SSL concept to high-temperature superconductors is thus related in a crucial way to the nature of the metal-insulator transition taking place for $\delta \rightarrow 0$ in two-dimensional systems. This is because the hole self-trapping competes with a spin liquid formation near the half-filled-band situation. On the other end, for small number of electrons, the Fermi liquid state must be stable as the interaction $\left(\sim n^{2}\right)$ becomes much smaller than the kinetic energy $(\sim n)$ of the particles. Hence the SSL state has a limited range of stability (if any). SSL state is realized in one-dimensional model systems for $n<1$, which are described by the Hubbard model [25]. We postulate the validity of the SSL 
concept also in two spatial dimensions.

To characterize quantitatively the statistical spin liquid one has to transform (2.1) to reciprocal $(k)$ space [4]. As a result one obtains

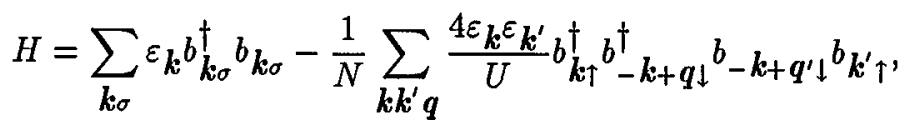

where $\varepsilon_{\boldsymbol{k}^{\prime}}$ is the bare band energy, and

$$
\begin{aligned}
& b_{k_{\sigma}}^{\dagger} \equiv \frac{1}{\sqrt{N}} \sum_{i} \exp \left(i \boldsymbol{k} \cdot \boldsymbol{R}_{i}\right)\left(a_{i \sigma}^{\dagger}-a_{i \sigma}^{\dagger} n_{i-\sigma}\right) \\
& =a_{\boldsymbol{k}_{\sigma}}^{\dagger}\left(1-n_{k_{-\sigma}}\right)+a_{\boldsymbol{k}_{\sigma}}^{\dagger} n_{\boldsymbol{k}-\sigma}-\frac{1}{N} \sum_{\boldsymbol{q} \boldsymbol{q}^{\prime}} a_{\boldsymbol{k}_{\boldsymbol{q}+\boldsymbol{q}-\boldsymbol{q}^{\prime} \sigma}}^{\dagger} a_{-\boldsymbol{q}^{\prime}-\sigma}^{\dagger} \boldsymbol{a}_{\boldsymbol{q}-\sigma} \\
& =a_{\boldsymbol{k}_{\sigma}}^{\dagger}\left(1-n_{k-\sigma}\right)+a_{\boldsymbol{k}_{\sigma}}^{\dagger} n_{\boldsymbol{k}-\sigma}+\frac{1}{N} \sum_{\boldsymbol{q}} a_{\boldsymbol{q}-\sigma}^{\dagger} S_{\boldsymbol{k}-\boldsymbol{q}}^{\sigma}
\end{aligned}
$$

with

$$
S_{p}^{\sigma}=\sum_{q} a_{p+q_{\sigma}}^{\dagger} a_{q-\sigma}
$$

being the Fourier component of the local spin operator $S_{i}^{\sigma} \equiv b_{i \sigma}^{\dagger} b_{i-\sigma}$. The operator $b_{k}^{\dagger}$ which describes the quasiparticle propagation in $k$ space is composed of three parts: (i) the projected fermion part $a_{k_{\sigma}}^{\dagger}\left(1-n_{k_{-\sigma}}\right)$; (ii) the part responsible for the Hubbard intersubband transitions $a_{k_{\sigma}}^{\dagger} n_{k_{-\sigma}}$ (this vanishes in the ground state configuration); and (iii) the spin-flip-associated-hopping part. Here, we are not interested in a detailed structure of the interaction between quasiparticles. This is because we are interested only in obtaining the simplest (mean-field-like) picture of the paired state, where the scattering processes are suppressed by binding into pairs and a subsequent formation of a condensed state. In such situation we take in the following $b_{k_{\sigma}}^{\dagger} \approx a_{k_{\sigma}}^{\dagger}\left(1-n_{k-\sigma}\right)$. Making this approximation and choosing only the pairing part with the resultant center-of-mass momenta $q=\boldsymbol{q}^{\prime}=0$ one obtains (2.5) in the form

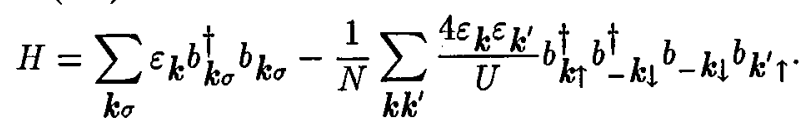

This Hamiltonian has the form of the BCS Hamiltonian, with a separable pairing potential $V_{k k^{\prime}}=-4 \varepsilon_{k^{\varepsilon}} \boldsymbol{k}^{\prime} / U$. However, the projected operators have nonfermion anticommutation relations

$$
\left\{b_{\boldsymbol{k}_{\sigma}}, b_{\boldsymbol{k}^{\prime} \sigma^{\prime}}^{\dagger}\right\}=\delta_{\boldsymbol{k} \boldsymbol{k}^{\prime}}\left\{\left(1-n_{\boldsymbol{k}-\sigma}\right) \delta_{\sigma-\sigma^{\prime}}+b_{\boldsymbol{k}-\sigma}^{\dagger} \boldsymbol{k}_{\boldsymbol{k} \sigma}\left(1-\delta_{\sigma-\sigma}\right)\right\}
$$

The nonfermion nature of the operators $b_{k_{\sigma}}$ and $b_{k_{\sigma}}^{\dagger}$ leads to principal differences with the BCS approach, as demonstrated below. For example, the Bogolyubov transformation diagonalizing (2.7) in the mean field (BCS) approximation is not applicable. Also, the normal properties will be different from those for a Fermi liquid, as one can show explicitly by calculating the statistical properties of 
the SSL in the normal state. In that state one can ignore the pairing part and solve the single-particle part utilizing the anticommutator Green-function formalism [26] to obtain the single-particle propagator in the form

$$
G_{k}(\omega) \equiv\left\langle\left\langle b_{k_{\sigma}} \mid b_{k_{\sigma}}^{\dagger}\right\rangle\right\rangle_{\omega}=\frac{1}{2 \pi} \frac{1-\tilde{n}_{k-\sigma}}{\varepsilon-\varepsilon_{k}}
$$

where $\tilde{n}_{k_{-s}} \equiv\left\langle a_{k_{\sigma}}^{\dagger} a_{k_{\sigma}}\right\rangle$. One notices two features. First, the spectral density function $A_{k}=1-\tilde{n}_{k-\sigma}$ is not constant and equal to unity. Second, by applying to $(2.8)$ the fluctuation-dissipation theorem one obtains the following distribution function:

$$
\left\langle b_{k \sigma}^{\dagger} b_{k \sigma}\right\rangle=\left(1-\tilde{n}_{k-\sigma}\right) f_{k \sigma},
$$

where $f_{k_{\sigma}}=\left\{1+\exp \left[\beta\left(\varepsilon_{k}-\mu\right)\right]\right\}^{-1}$ is the Fermi-Dirac distribution. Explicitly [3]

$$
\left\langle b_{k_{\sigma}}^{\dagger} b_{k_{\sigma}}\right\rangle=\frac{1}{2} \frac{1}{1+(1 / 2) \exp \left[\beta\left(\varepsilon_{k}-\mu\right)\right]} \text {. }
$$

This is a correct Fermi-Dirac distribution, in which the first factor (1/2) expressed the projection of double occupancies $|k \uparrow \downarrow\rangle$ and, hence, the reduction by half of the number of available states. The second factor $(1 / 2)$ leads to the linear in $T$ renormalization of the chemical potential $\mu \rightarrow \tilde{\mu}=\mu+k_{\mathrm{B}} T \ln 2$, if we rewrite the large fraction part in (2.9) as in the Fermi-Dirac form. This linear part in $\mu=\mu(T)$ is absent for ordinary fermions and has two far reaching consequences in the following sense. Firstly, the reduction by half of the available $|k \sigma\rangle$ quasiparticle states in the subband leads to a filled-band configuration for $n=1$, which now represents the Mott insulating state. This filled-band state can become magnetic since double occupancies are excluded in this representation. In fact, a stable antiferromagnetic phase is obtained close to the half filling, as discussed in a separate contribution to this conference [27].

Secondly, one can ask the question whether the spin liquid concept is in agreement with the spectacular linear in temperature behavior of resistivity in a wide range of temperature and for different high temperature superconductors. It is well known [28] that the relaxation time $\tau$ depends linearly on both $T$ and quasiparticle energy $\varepsilon$. It is therefore natural to assume that

$$
\tau(\varepsilon)=A \frac{h}{|\varepsilon-\mu|+a k_{\mathrm{B}} T},
$$

where $A$ and $a$ are dimensionless constants. Next, assuming that the relaxation-time approximation is valid one obtains the following expression for the static conductivity [29]:

$$
\sigma(T) \equiv \int \sigma(E)\left(-\frac{\partial \tilde{f}}{\partial E}\right) \mathrm{d} E
$$

with $\sigma(\varepsilon) \sim \tau(\mu)$, where $\tilde{f}(E)$ is the Fermi-Dirac distribution with the chemical potential replaced by $\tilde{\mu}$. Making the low-temperature expansion for (2.11), a straightforward analysis leads to the temperature dependence of the electrical conductivity

$$
\sigma(T)=\frac{e^{2}}{h a_{0}} \frac{A}{2 k_{\mathrm{B}} T(a+\ln 2)}\left(1+\sum_{n=1}^{\infty}\left(2^{2 n}-1\right) \frac{\pi^{2 n} B_{n}}{(\ln 2+a)^{2 n}}\right),
$$


where $a_{0}$ is the lattice constant, and $B_{n}$ are the Bernoulli numbers. We see that $\sigma^{-1}=\rho \sim T$ in all orders provided the series is convergent. The above argument is by no means a proof of the linear $(\rho \sim T)$ resistivity. It merely means that the spin liquid concept is not in contradiction with the experiment. The heart of the problem is thus reduced to a microscopic derivation of Eq. (2.10), the procedure which should determine microscopically the constants $A$ and $a$.

The present treatment of the statistical spin liquid starts from a treatment of electrons as independent particles, with the dispersion relation $\varepsilon_{k}$. It does not contain any band narrowing factor due to electron correlations. Nonetheless, a straightforward calculation of the band energy $E_{\mathrm{B}}$ of fermions in SSL state yields for the featureless (rectangular) form of the density of states the result

$$
E_{\mathrm{B}} / N=-(W / 2) n(1-n),
$$

where $W$ is the bare bandwidth. In the limit $n \rightarrow 1$ the band energy vanishes and only the kinetic exchange part in (2.9) survives, which leads again to the antiferromagnetic insulating ordering as a reference state. The quantity $W$ is a measure of the spread of the $3 d$ states in $\mathrm{CuO}_{2}$ planes, detected in the corresponding photoemission data.

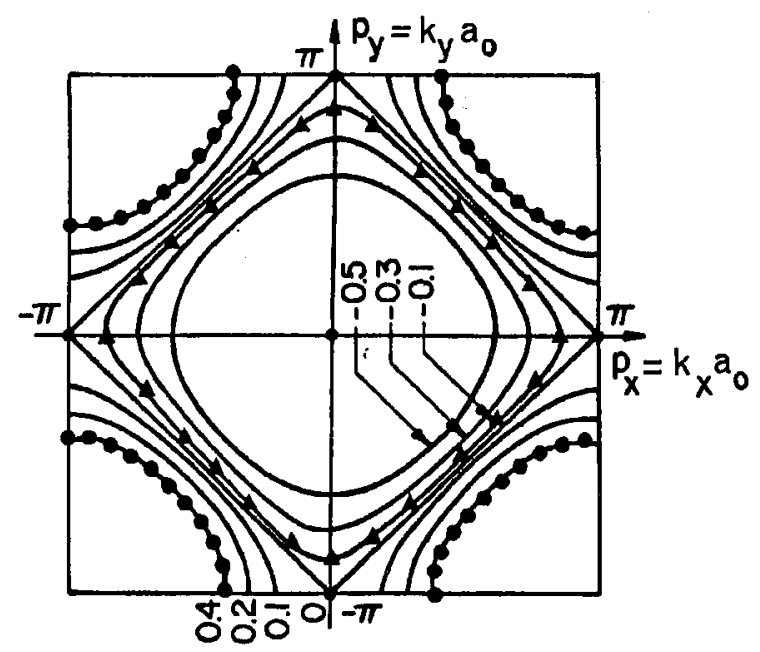

Fig. 1. The Fermi surface for the square lattice with number of electrons per atom $n<1$ in case of Fermi liquid (triangles) and spin liquid (full circles). The specified values of the Fermi energy are in units of bare bandwidth $W$.

Recent photoemission experiments [30] determined the shape of the Fermi surface (FS). In Fig. 1 we have presentedthe shape of the Fermi surface for a 
two-dimensional Fermi liquid with $n<1$ for a square lattice (triangles), and for the same filling in the spin-liquid case (full circles). The hole-like Fermi surface for the SSL case agrees with the observed results. Our very recent analysis of the three-orbital model [31] is also in qualitative agreement with the observations. Nonetheless, a careful quantitative analysis of the data is required and will be reported as the detailed data of Campuzano et al. [30] become available to us.

\subsection{Superconducting state of a statistical spin liquid}

In this section we summarize the results for a paired state of the BCS type. We start from (2.7) as the effective Hamiltonian with pairing induced by the kinetic exchange. We also assume that the statistical distribution of electrons is governed by (2.9). Concrete results are obtained for a square lattice.

A single remark concerning one specific feature of the pairing is in order here. The real space pairing bears its origin from virtual intersite hopping processes with doubly occupied site configuration in the intermediate state. In this respect, the present pairing differs from that induced by an exchange of a Bose quasiparticle, which contains retardation by its nature. Also, the real space pairing differs from that induced by the Coulomb interaction and involving plasmon exchange, because the latter is induced by its long-range part and engages a quasiparticle.

To solve model Hamiltonian (2.7) for the superconducting phase one can employ the Green function formalism [26] in the Nambu representation. In that formalism the approximation equivalent to the BCS approach is achieved by defining the Nambu operator $A_{k}^{\dagger}=\left(b_{k \uparrow}, b_{-k_{\downarrow}}^{\dagger}\right)$ and postulating the mean-field equation in the form

$$
\mathrm{i} \frac{\mathrm{d}}{\mathrm{d} t} \boldsymbol{A}_{\boldsymbol{k}}=\boldsymbol{M A _ { k }}
$$

where $M$ is $2 \times 2$ matrix, which is calculated explicitly elsewhere [32]. Here we only summarize the results and discuss their physical meaning. These results can be discussed and the following headings:

1 . The self-consistent equation apart from a statistical factor $\left(1-\tilde{n}_{k} / 2\right)$ has the BCS form [12]. The quasiparticle energy is as in the BCS theory, with the gap in the form

$$
\Delta_{k}=\left(1-\tilde{n}_{\boldsymbol{k}} / 2\right) \Delta(T) \varepsilon_{\boldsymbol{k}}
$$

where $\Delta(T)$ is the gap parameter. The statistical factor $\left(1-\tilde{n}_{k} / 2\right)$ will reduce the value of the gap by half below the Fermi surface, whereas the factor $\varepsilon_{k}$ leads to the solution of an extended s-wave symmetry, since for a square lattice $\varepsilon_{k} \sim \cos \left(k_{x} a_{0}\right)+\cos \left(k_{y} a_{0}\right)$.

The extended $s$-wave symmetry of $\Delta_{k}$ is caused by the circumstance that we have included the three-site terms in the kinetic-exchange part in (2.1). If the three-site part was neglected, then the $d$-wave solution would appear as a possibility at intermediate hole concentration. Very recent experiments [33] suggest the $d$-wave as the correct symmetry of the superconducting order parameter, although the situation is not as yet settled. From the theoretical point of view, the situation calls for a careful analysis, whether the derivation [8] of the effective Hamiltonian 
(2.1) from the Hubbard Hamiltonian, which produces the three-site term, is equivalent to the derivation of $t-J$ model [34], which starts from a multiorbital model reduced subsequently to a single-band case.

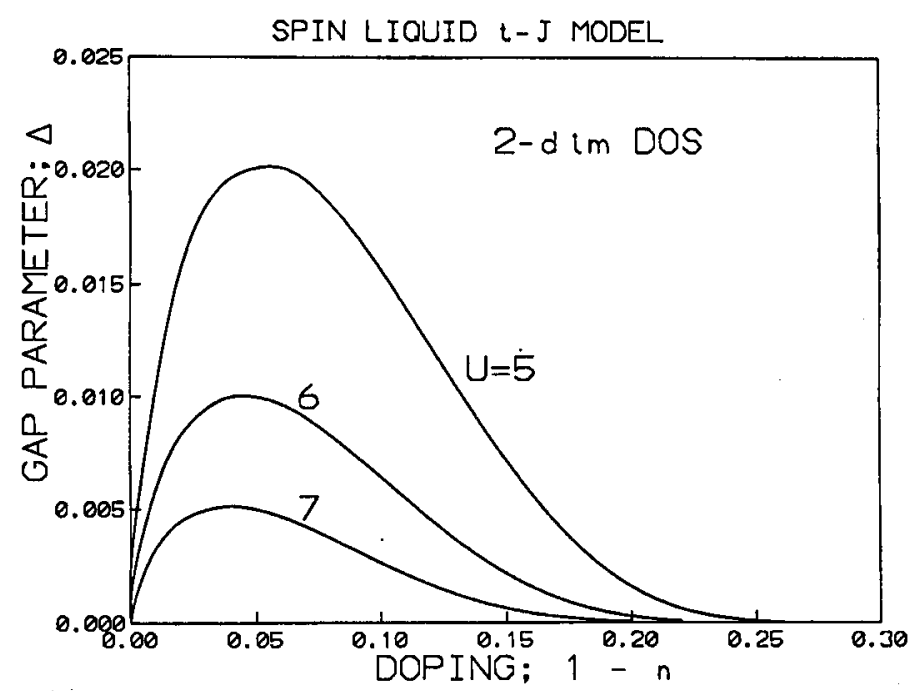

Fig. 2. Superconducting gap parameter $\Delta(0)$ as a function of the hole number $\delta=1-n$, for different values of $U$ measured in units of $2|t|=W / 4$.

In Fig. 2 we display the parameter $\Delta(T)$ for $T=0$ as a function of $\delta=1-n$. Similar results are obtained [32] for the value of $T_{\mathrm{c}}$. The superconductivity vanishes both for $n \rightarrow 1$ and for $n \geq 0.15 \div 0.25$, depending on the value of $U$. This character is in agreement with experiment for $\mathrm{La}_{2-x} \mathrm{Sr}_{x} \mathrm{CuO}_{4}$. A similar solution is obtained in the regime $0<n<0.15 \div 0.25$, reflecting the hole-electron symmetry for the statistical spin liquid. It is interesting to ask if the latter solution reflects the situation in $n$-type superconductors $\mathrm{Nd}_{2-x} \mathrm{Ce}_{x} \mathrm{CuO}_{4}$. We have no answer as yet to that question.

2. The average occupation number $\tilde{n}_{k \sigma}$ for SSL will differ from that for the Fermi fluid, both in normal and superconducting phases. This is because the states $|k \uparrow \downarrow\rangle$ are excluded from the physical space and therefore, the Fermi level is placed higher inside the band than the position for the same concentration in the FL case. This difference in position of the chemical potential is directly related to the Luttinger theorem violation. In connection with this one should mention the claim $[30,33]$ that the measured Fermi surface topology is in agreement with LDA band-structure calculations [35]. However, in order to make experiment and the LDA theoretical results to agree, both the Fermi velocity and the effective mass must be renormalized by a factor of two [30]. The problem of applicability or inapplicability of the spin-liquid concept to the planar electron should be resolved in the near future. 
3. The representation of high-temperature superconductivity as a paired state for a planar spin liquid is unsufficient because of a number of reasons. Firstly, the superconductivity encountered in high-temperature materials as a three-dimensional phenomenon. Therefore, in order to reconcile the two-dimensional metallicity in the normal phase with three-dimensional superconducting state one has to introduce an interplanar Cooper-pair hopping; such processes are enhanced with respect to the single-electron hopping because this process lowers additionally the system energy relative to the normal state.

Secondly, when one compares the strength of the pairing coupling constant $J=W^{2} / z U$, where $z=4$ is the coordination number, with the spin-liquid energy per site, which is $-(W / 2)(1-n)$, one sees that $J>(W / 2) \delta$ for $n<n_{\mathrm{c}}$, where the critical value is $n_{\mathrm{c}}=2 / U \sim 0.2-0.25$. This means that in the whole superconducting range of hole concentration the coupling constant should be regarded as large. In other words, strong-coupling effects may be important in the analysis of a planar superconductivity for a statistical spin liquid. The same should hold true also for a Fermi liquid approach as the expressions for the band energy in both SSL and FL cases coincide (the latter result is obtained in the Gutzwiller approximation).

The reasoning above provides also a very simple and clear interpretation of the binding induced by the real space pairing $\sim t^{2} / U$. Namely, the kinetic energy of particle in the spin liquid is reduced by the factor $(1-n) /(1-n / 2) \approx 2 \delta$ relative to independent particle motion. Therefore, the time for a hole to stand still is $\tau_{\mathrm{B}} \sim \tau_{\mathrm{B}}^{0} / 2 \delta$, where $\tau_{\mathrm{B}}^{0}$ is the Heisenberg time uncertainty for a band to form: $\tau_{\mathbf{B}}^{0} \approx h / W$. On the other hand, the time uncertainty for the electron pair to undergo a virtual hopping leading to the kinetic exchange is $\tau_{\mathrm{ex}} \approx h / J=h U z / W^{2}$. In the whole superconducting regime $\tau_{\mathrm{ex}} \geq \tau_{\mathrm{B}}$ and hence the electron neighboring partner binds to it before it can escape. The two time scales become comparable for $n \approx n_{c}$. In other words, in the normal state $\left(\delta>\delta_{c}\right)$ the single hole dynamics is predominant and causes the dissociation of the pairs bound by the short-range interaction in direct space. This dissociation would be very difficult to understand within the pairing operating in the reciprocal space only.

\section{Real space pairing for heavy fermions: the Kondo exchange}

The high temperature superconductors (HTS) and heavy fermion (HF) systems represent probably the materials, which exhibit spectacular electron correlation effects. Both materials represent compounds in which the active correlated states ( $3 d$ and $4 f$ or $5 f$ states, respectively) are hybridized with magnetically inert $(2 p$ and $d-s)$ states. The question is then what is the difference between the two classes of materials? This difference is represented schematically in Fig. 3. Namely, the heavy fermion limit represents an asymmetric Anderson-lattice case, in which the bare atomic $f$ level is placed close to the Fermi level. On the contrary, the $3 d^{8}$ and $3 d^{10}$ levels in high-temperature superconductors are placed almost symmetrically with respect to the bare Fermi level in the $2 p$ band (by "bare" we understand the level and band positions before the hybridization $V$ and the $f-f$ or $d-d$ intraatomic Coulomb interaction $U$ is taken into consideration). Additionally, the 


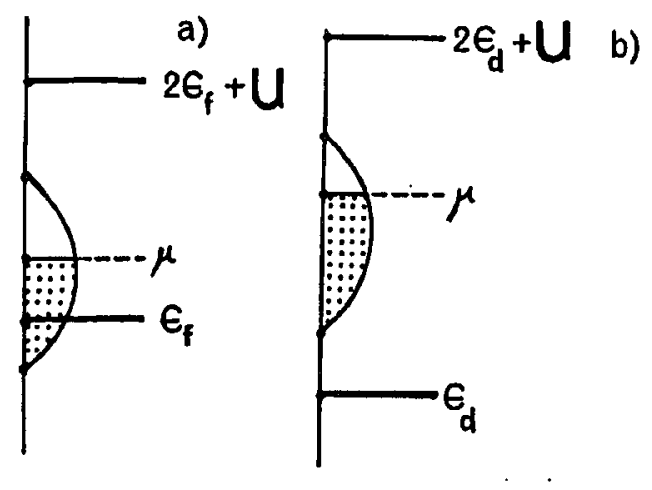

Fig. 3. Schematic representation of the electronic structure of a heavy-fermion system (a) and a high-temperature superconductor (b).

ratio $|V| /\left(\varepsilon_{f}-\mu\right)$ is $\ll 1$ and $\approx 1$ for HF materials and HTS, respectively. Therefore, in the HF systems the Kondo coupling is rather small and can participate in the quasiparticle dynamics, whereas it is strong in HTS and in conjunction with a symmetric position of $3 d^{8}$ and $3 d^{10}$ levels leads to an effective single band representation of the dynamics [34].

In order to derive the explicit form of the Anderson Hamiltonian a division of hybridization processes into low- and high-energy parts is necessary [10]. In effect, one obtains the following effective Hamiltonian with the real space pairing in the lowest order in $1 / U$ :

$$
\begin{aligned}
H= & \sum_{\boldsymbol{k}_{\sigma}} \varepsilon_{k} n_{k \sigma}+\varepsilon_{f} \sum_{i \sigma} b_{i \sigma}^{\dagger} b_{i \sigma}+\sum_{i m \sigma}\left(V_{i m} b_{i \sigma}^{\dagger} c_{m \sigma}+\text { h.c. }\right) \\
& -\sum_{i m n} \frac{2 V_{m i}^{*} V_{i n}}{U} \tilde{B}_{i m}^{\dagger} \tilde{B}_{i n} .
\end{aligned}
$$

The first term represents now the band energy of uncorrelated electrons. The second term expresses the energy of localized correlated electrons. The third term describes the hybridization between the two subsystems, whereas the last provides real space pairing between the two subsystems, with

$$
\tilde{B}_{i m}^{\dagger} \equiv \frac{1}{\sqrt{2}}\left(b_{i \uparrow}^{\dagger} c_{m \downarrow}^{\dagger}-b_{i \downarrow}^{\dagger} c_{m \uparrow}^{\dagger}\right)
$$

being the pair creation operator of $\left|f_{i} \uparrow\right\rangle$ state and the conduction $\left|c_{m} \downarrow\right\rangle$ state. The value $\tilde{U}$ is $U+\varepsilon_{f}$, and $\varepsilon_{f}$ is the bare $f$-level position.

An explicit solution of the model (3.2) in the mean-field approximation and using the functional integration technique over the coherent Fermi and Bose fields, has been discussed in a separate contribution to this conference [36]. In that approach the slave-boson representation [37] $b_{i \sigma}^{\dagger} \equiv e_{i} f_{i \sigma}^{\dagger}$ is used, where $e_{i}$ is boson annihilation operator and $f_{i \sigma}$ is the same operator for a pseudofermions. Here, we underline some specific features of the normal and superconducting phases, namely: 
1. The heavy-fermion FL state is formed if the condition $\left|\varepsilon_{f}-\mu\right| / \rho_{0} V^{2} \gg 1$ is met, where $\rho_{0}$ is the density of states at the Fermi level in the bare band. The quasiparticle picture is such that the effective position $\varepsilon_{f}$ of the $f$ level is very close to the Fermi level, i.e. $\tilde{\varepsilon}_{f}-\mu \equiv k_{\mathrm{B}} T_{\mathrm{K}}$, where $T_{\mathrm{K}}$ is a new energy scale for the problem, the so-called effective Kondo temperature. The density of quasiparticle states at the Fermi level is $\rho(\mu)=1 / 2 k_{\mathrm{B}} T_{\mathrm{K}}$ and is proportional to the effective mass. The overall width of the quasiparticle band is $D=\left(2 k_{\mathrm{B}} T_{\mathrm{K}} W\right)^{1 / 2} \gg k_{\mathrm{B}} T_{\mathrm{K}}$.

2. The superconducting gap for $\Delta_{k}$ has the same zeros as the Fourier transform $V_{k}$ of the hybridization matrix element $V_{i m}$. In other words, the points $k$ for which the gap vanishes are determined by the point symmetry of the $f$ atom in the intermetallic heavy-fermion compound. A classification of the order-parameter symmetries on the basis of point-group representations is under way [38].

3. The superconducting state disappears in the limit of integral occupancy of the $f$ level $\left(n_{f} \rightarrow 1\right)$. This means that the Kondo coupling $\sim U^{-1}$ describes indeed the superconducting state which disappears in the true Kondo-lattice regime, when the $f$ moments become localized.

At this point a basic question should be raised. Namely, what is the reference state for the heavy-fermion systems, which would correspond to the antiferromagnetic insulator for the high-temperature superconductors? Is it a magnetic metal (viz. a rare-earth metal), or is it a Kondo-lattice state, with the localized $f$ moments, which are coupled antiferromagnetically to the abundant conduction electrons? Part of the difficulty in understanding the heavy-fermion behavior is connected with the missing knowledge of the reference state.

4. The basic superconducting properties scale in a universal manner with $T_{\mathrm{K}}$, as discussed elsewhere $[36,39]$.

At the end of this section we would like to address the following question: why the heavy-fermion systems can be represented by a Fermi liquid state and the high-temperature superconductors cannot? There are two reasons for that. First, HF materials are anisotropic (viz. magnetic susceptibility in $\mathrm{UPt}_{3}$ ), but truly 3-dimensional systems in the normal state, while HTS are not. Second, the proximity of the $f$ level to the Fermi level in HF systems induces in a substantial conduction-band state admixture to the $f$ state, resulting in an essential reduction of the effective Coulomb interaction $U$. Such reduction seems to be ineffective in quasi-two-dimensional systems.

\section{Conclusions}

We have addressed some of the principal questions concerning:the nature of electronic states in the two systems mentioned in the title, as well as have summarized the concept of real space pairing $[2,14]$ induced by the full form (with three-site terms) of the kinetic exchange interaction in the first nontrivial order in $1 / U$. We have also discussed briefly the consequences of the proposed pairing on the mean-field level. The most fundamental feature of the presented approach is that it is the only model in which the superconductivity and antiferromagnetism (or other spin-singlet type of state) are intimately connected, even if their coexistence regime is practically nonexistent, as in the HTS case. Further work is needed 
to incorporate the observed magnetic moment localization into a coherent picture of correlated fermion states in those fascinating materials, which represent a practical realization of a unification of physics of antiferromagnetic semiconductors, superconducting metals, as well as of the transition from a magnetic insulator to superconducting metal.

The author acknowledges the cooperation, numerous discussions and technical help of his coworkers: W. Wójcik, K. Byczuk and J. Karbowski. The work was supported by the Committee for Scientific Research (Poland), grants Nos. 204299101 and 2 P302 093 05, and by MISCON, grant No. DE-FG 02-90ER 45427. He is also indebted to Profs. J.M. Honig and A.W. Overhauser from Purdue University for discussions and a constant encouragement.

\section{References}

[1] For review see K. Levin, Ju H. Kim, J.P. Lu, Qimiao Si, Physica C 175, 449 (1991).

[2] P.W. Anderson, in: Frontiers and Borderlines in Many-Particle Physics, Eds. R.A. Broglia, J.R. Schrieffer, North-Holland, Amsterdam 1988, p. 1.

[3] J. Spałek, W. Wójcik, Phys. Rev. B 37, 1532 (1988).

[4] J. Spałek, Phys. Rev. B 40, 5180 (1989).

[5] J. Spałek, Physica B 163, 621 (1990).

[6] J. Spałek, W. Wöjcik, Sci. Bull. Jagellonian University 29, 87 (1990).

[7] For review see: J. Spalek, K. Byczuk, J. Karbowski, W. Wójcik, Phys. Scr., in press.

[8] K.A. Chao, J. Spałek, A.M. Oles', J. Phys. C 10, L271 (1977); Phys. Rev. B 18, 3453 (1978).

[9] P.W. Anderson, Phys. Rev. 115, 2 (1959).

[10] J. Spałek, Phys. Rev. B 38, 208 (1988).

[11] J.R. Schrieffer, P.A. Wolff, Phys. Rev. 149, 491 (1966); J. Spatek, A.M. Oleś, K.A. Chao, Phys. Status Solidi B 87, 625 (1978); Phys. Rev. B 18, 3748 (1978).

[12] K. Byczuk, J. Spalek, proceedings of this conference, part II.

[13] P.W. Anderson, Science 235, 1196 (1987).

[14] J. Spalek, Phys. Rev. 37, 533 (1988).

[15] S. Chakraverty, B.I. IIalperin, D.R. Nelson, Phys. Rev. Lett. 60, 1057 (1988).

[16] For review see J.M. Tranguada, in: Earlier and Recent Aspects of Superconductivity, Eds. J.G. Bednorz, K.A. Müller, Springer, Berlin 1990, p. 422.

[17] B.I. Shraiman, E.D. Siggia, Phys. Rev. Lett. 60, 740 (1988); S.A. Trugman, Phys. Rev. B 37, 1597 (1988).

[18] A.F. Ruckenstein, P.J. Hirschfeld, J. Appel, Phys. Rev. B 36, 857 (1987).

[19] J. Hubbard, Proc. R. Soc. Lond. A 281, 401 (1964).

[20] E. Lieb, F.Y. Wu, Phys. Rev. Leit. 20, 1445 (1968).

[21] V. Dobrosavljević, G. Kotliar, Acta Phys. Pol. A 85, 21 (1994).

[22] J. Spałek, A. Datta, J.M. Honig, Phys. Rev. Lett. 59, 728 (1987).

[23] N.F. Mott, Metal-Insulator Transitions, Taylor \&Francis, London 1974.

[24] P.W. Anderson, Phys. Rev. Lett. 65, 2306 (1990). 
[25] J.B. Sokoloff, Phys. Rev. B 2, 779 (1970).

[26] D.N. Zubariev, Usp. Fiz. Nauk 71, 71 (1960).

[27] J. Spałek, W. Wójcik, proceedings of this conference, Part II.

[28] For review see P.W. Anderson, Physica $C$ 185-189, 11 (1991).

[29] See e.g. N.F. Mott, H. Jones, The Theory of the Properties of Metals and Alloys, Clarendon Press, Oxford 1936, Ch. 7.

[30] J.C. Campuzano, G. Jennings, M. Faiz, L. Beaulaigue, B.W. Veal, J.Z. Liu, A.P. Paulikas, K. Vandervoort, H. Claus, R.S. List, A.J. Arko, R.J. Bartlett, Phys. Rev. Lett. 64, 2308 (1990), and private communication.

[31] K. Byczuk, J. Spałek, unpublished.

[32] K. Byczuk, J. Spałek, submitted to Phys. Rev. B.

[33] For an overview see Physics Today, May 1993; Z.X. Shen, private communication.

[34] F.C. Zhang, T.M. Rice, Phys. Rev. B 37, 3759 (1988).

[35] J. Yu, S. Massidda, A.J. Freeman, D.D. Koeling, Phys. Lett. A 122, 203 (1987).

[36] J. Karbowski, J. Spałek, proceedings of this conference, part II.

[37] A.J. Mills, P.A. Lee, Phys. Rev. B 35, 3394 (1987).

[38] J. Karbowski, private communication.

[39] J. Karbowski, J. Spałek, submitted to Phys. Rev. B. 